\title{
Fatores que interferem no ensino e aprendizagem de residentes multiprofissionais em saúde: revisão integrativa
}

\author{
Factors interfering in teaching and learning of multiprofessional \\ residents in health: integrative review
}

Amanda de Lemos Mello ${ }^{1,2}$, Guilherme Tavares de Arruda ${ }^{3}$, Marlene Gomes Terra ${ }^{4}$, Cristiane Trivisiol Arnemann ${ }^{4}$, Daiana Foggiato de Siqueira ${ }^{5}$

'Programa de Pós-Graduação em Enfermagem, Universidade Federal de Santa Maria (UFSM) - Santa Maria (RS), Brasil. Departamento de Enfermagem, UFSM - Santa Maria (RS), Brasil.

${ }^{3}$ Curso de Fisioterapia, UFSM - Santa Maria (RS), Brasil.

${ }^{4}$ Curso Técnico em Enfermagem, Colégio Politécnico, UFSM - Santa Maria (RS), Brasil.

Departamento de Enfermagem, Universidade Regional Integrada do Alto Uruguai e das Missões de Santiago (URI) - Santiago (RS), Brasil.

DOI: https://dx.doi.org/10.7322/abcshs.v44i2.1176

\section{RESUMO}

Os Programas de Residência Multiprofissionais em Saúde estão em plena expansão no território brasileiro, tornandose uma estratégia de formação em saúde que busca o fortalecimento da qualidade da atenção à saúde da população. Esta revisão objetivou identificar as evidências disponíveis nos artigos científicos sobre os fatores que interferem no ensino e aprendizagem de residentes multiprofissionais em saúde. Tratase de uma revisão integrativa de literatura desenvolvida nas bases de dados LILACS, Medline e Scopus, na qual foram incluídos 35 estudos, a partir de palavras-chave e descritores, no período de 2005 a 2017. Nos resultados evidenciou-se fatores relacionados à compreensão do papel do residente; a importância da formação dos apoiadores pedagógicos e a interação entre as profissões; a pactuação das atividades entre as instituições de ensino e serviço; a relação entre a teoria e a prática e o desenvolvimento das ações de Educação Permanente em Saúde; a formulação das estratégias de aprendizagem, do planejamento e execução dos espaços formativos; e, os processos avaliativos. Em conclusão o estudo possibilitou identificar os fatores que interferem no ensino e aprendizagem de residentes multiprofissionais em saúde, os quais perpassam por aspectos da construção do aprender e fazer no trabalho coletivo, da integração ensino-serviço e da organização didática-pedagógica.

Palavras-chave: internato não médico; capacitação em serviço; desenvolvimento de pessoal; educação superior.

\begin{abstract}
Multiprofessional Health Residency Programs are in full expansion in Brazil, becoming a health training strategy that seeks to strengthen the quality of health care for the population. This review aims to identify available scientific articles about the factors that interfere in the teaching and learning of residents in multiprofessional health. This is an integrative review of the literature from the databases LILACS, Medline and Scopus, which included 35 studies, using defined key words and descriptors, during the period of 2005 through 2017 . The results revealed factors related to the understanding of the resident role; the importance of the training of the pedagogical supporters and the interaction among professionals; the pacing of activities between the teaching and health facility; the relation between theory and practice and the development of the actions of Permanent Education in Health; the formulation of learning strategies; planning and execution in training spaces; and the evaluation processes. In conclusion the study made possible to understand the factors that interfere in teaching and learning of multiprofessional residents in health, which involve aspects the learning construction and practice in collective work, teaching-service integration and pedagogical organization.
\end{abstract}

Keywords: internship nonmedical; inservice training; staff development; education higher.

Recebido em: 11/06/2018

Revisado em: 29/11/2018

Aprovado em: $17 / 12 / 2018$

Autor para correspondência: Amanda de Lemos Mello - Universidade Federal de Santa Maria - Avenida Roraima, 1000, prédio 26, sala 1445 -

CEP: 97105-900 - Santa Maria (RS), Brasil - E-mail: amandamello6@yahoo.com

Conflito de interesses: nada a declarar. 


\section{INTRODUÇÃO}

O debate referente ao planejamento das ações voltadas à formação dos profissionais da área da saúde, desde sua primeira referência em 1958, pela publicação da Organização Pan-Americana de Saúde (OPAS) e da Organização Mundial da Saúde (OMS), passa a ser difundido como uma prioridade. Com a criação do Sistema Único de Saúde (SUS), essas ações são regulamentadas, com vistas a propor novas relações no trabalho pautadas no papel estratégico dos profissionais no desenvolvimento do SUS, como o desenvolvimento em âmbito nacional dos Programas de Residência Multiprofissional em Saúde.

Por meio de parcerias firmadas entre o Ministério da Saúde e o Ministério da Educação, as Residências Multiprofissionais em Saúde (RMS) foram regulamentadas pela Lei Federal no 11.129 de 2005 e regidas como Pós-graduação Lato Sensu. Estas têm como objetivo promover a qualificação de profissionais para o SUS por meio de práticas pedagógicas orientadas pelos princípios da Educação Permanente em Saúde (EPS) e desencadeando espaços em que atores dialogam entre $\mathrm{si}^{1}$.

As RMS emergem como uma possibilidade concreta de problematização da realidade e articulação das instituições de ensino, serviço de saúde e comunidade, integrando residentes, docentes, usuários, gestores, trabalhadores e profissionais de saúde no cotidiano dos serviços e de acordo com a realidade regional. Além disso, os Programas de Residência Multiprofissional em Saúde (PRMS) tendem a permitir a permeabilidade das ações educativas no cotidiano das práticas de saúde, vislumbrando um novo modelo de gestão e atenção à saúde, por meio do desenvolvimento da EPS, aos profissionais vinculados aos serviços de saúde ${ }^{1,2}$.

Sob essa perspectiva, a qualidade do processo formativo, respondendo à necessidade de um perfil profissional inovador e comprometido com a realidade da saúde da população, é um ponto a ser frequentemente repensado, revisitado e reformulado. Uma das estratégias para colaborar com qualidade do ensino e aprendizagem RMS é reflexionar frente às evidências cientificas, pois, existem desafios quanto à organização e estrutura destes programas, os quais podem interferir em seu processo ${ }^{3}$.

Compreende-se que, com a crescente quantitativa destes programas em âmbito brasileiro, tem-se uma preocupação ascendente da qualidade da formação destes profissionais. Visto isto, faz-se premente identificar fatores que podem vir a interferir neste processo de formação pode auxiliar gestores, residentes, tutores e preceptores das instituições de ensino e dos serviços de saúde.

A importância dos Programas de Residência Multiprofissional em Saúde (PRMS) para o fortalecimento do SUS e a ampliação da assistência em saúde já vem sendo evidenciada em diversos estudos atuais ${ }^{1-4}$. No entanto, apresentam-se, também, desafios a serem fortalecidos no contexto da formação destes Programas de Residência.
Com base neste contexto, este estudo tem como objetivo identificar as evidências disponíveis nos artigos científicos sobre os fatores que interferem no ensino e aprendizagem de residentes multiprofissionais em saúde.

\section{MÉTODOS}

Trata-se de um estudo de revisão integrativa da literatura guiado por seis etapas. Na primeira etapa do estudo, foi elencada a questão de pesquisa: quais os fatores que interferem no ensino e aprendizagem de residentes multiprofissionais em saúde? A busca foi desenvolvida no mês de janeiro de 2018, por dois pesquisadores de forma independente, nas bases de dados eletrônicas Literatura Latino-Americana e do Caribe em Ciências da Saúde (LILACS), na Public Medical Literature Analysis and Retrieval System Online (PubMed) e ScriVerse Scopus (SCOPUS), compondo a segunda etapa do estudo.

A estratégia de busca adotada para esta revisão foi a partir da combinação entre palavras chave e do descritor no idioma português, o qual foi extraído do Banco de Descritores em Ciências da Saúde (DeCS): "internato não medico" [Descritor de assunto] or "residência" and "multiprofissional" [Palavras]" e no idioma inglês, extraído do Medical Subject Headings (MeSH): "Internship Nonmedical" [MeSH terms] or "Internship" and "multiprofessional" [Keyword]. Justifica-se a escolha do descritor "internato não médico", pois trata-se de um descritor utilizado por pesquisadores da temática em suas produções, sendo considerado o descritor que mais se aproxima das produções científicas acerca das residências multiprofissionais em saúde. Além do mais, após tentativas de uso de descritores, justifica-se a utilização das palavras chave devido a possibilidade de abarcar maior números de estudos que pudessem responder ao objeto de pesquisa.

$\mathrm{Na}$ terceira etapa da pesquisa, foram estabelecidos os critérios de inclusão: artigos originais de pesquisa que tivessem como tema central a formação em programas de residência multiprofissional em saúde; nos idiomas português, inglês ou espanhol, publicados no período de 2005 a 2017, uma vez que o marco inicial está definido pela Lei Federal 11.129/2005, que institui os Programas de Residência Multiprofissional em Saúde no âmbito brasileiro. E, como critérios de exclusão: artigos que obtivessem como objetos de estudo apenas uma área profissional dos programas de residências.

A busca totalizou 1.244 produções que compõe o cenário global das publicações, as quais foram selecionadas por meio da leitura dos títulos e resumos, sendo submetidas aos critérios estabelecidos. Os artigos repetidos foram analisados e contabilizados somente uma vez, sendo que resultaram 35 artigos primários ${ }^{1,3,5-37}$ a serem analisados na íntegra (Figura 1). Vale ressaltar que a maioria dos estudos excluídos pela temática tratava de residência uniprofissional, ou seja, apenas uma área profissional. Além 
disto, esta revisão integrativa priorizou os estudos desenvolvidos em âmbito brasileiro a fim de ir ao encontro da Lei Federal 11.129/2005, que defini o desenvolvimento dos Programas de Residência Multiprofissional (Figura 1).

Para análise e posterior síntese dos resultados dos artigos selecionados para leitura na íntegra, buscou-se realizar a leitura dos artigos por dois pesquisadores isoladamente com a finalidade de minimizar a possibilidade de viés na seleção dos estudos. $\mathrm{Na}$ quarta etapa, foram elencadas as informações que seriam extraídas dos artigos publicados. Para isso, foi utilizado um quadro sinóptico construído para esse fim, contemplando aspectos gerais dos artigos publicados (procedência do primeiro autor; delineamento de pesquisa; nível de evidência), bem como os principais resultados separados didaticamente em fatores que interferem no ensino e aprendizagem.

As forças de evidência dos estudos foram avaliadas de acordo com a classificação de evidências para diferentes questões clínicas que, dependendo da questão do estudo primário, é necessário utilizar uma hierarquia de evidências (por níveis, sendo o nível 1 o mais forte), organizado da seguinte forma: tratamento/intervenção (CI); diagnóstico (CII); - 7 níveis/cada; prognóstico ou etiologia (CIII); significado ou experiência de doença ou a compreensão dos sentimentos do paciente sobre os efeitos de uma intervenção (CIV) - 5 níveis/cada ${ }^{38}$.

$\mathrm{Na}$ quinta etapa da pesquisa, os resultados foram alcançados após extração e interpretação das informações obtidas na etapa anterior do estudo. A apresentação dos resultados foi feita de forma descritiva, com vistas a possibilitar ao leitor a avaliação da finalidade da revisão integrativa elaborada, de forma a atingir a sexta etapa, ou seja, apresentada a síntese do conhecimento extraído acerca dos fatores que influenciam no ensino-aprendizagem de residentes multiprofissionais em saúde. Ressalta-se que foram respeitadas as dimensões éticas no que tange as ideias, os conceitos e as definições utilizados pelos autores dos artigos analisados.

\section{RESULTADOS}

O corpus de análise foi composto por 35 estudos primá$\operatorname{rios}^{1,3,5-37}$, dos quais $37,14 \%$ são procedentes da região sudeste do Brasil $(n=13)$. A maioria dos estudos foram desenvolvidos com delineamento qualitativo, $(\mathrm{n}=28,80 \%)$, a partir de questões clínicas voltadas a significados/experiências ( $\mathrm{n}=30,85,71 \%)$ (Tabela 1). A interpretação dos dados coletados possibilitou analisar as evidências dos estudos segundo as afinidades temáticas, conforme mostra a Tabela 2, que apresenta a síntese dos resultados encontrados (Tabela 2 ).

Os resultados desta revisão evidenciaram que os fatores que interferem no ensino e aprendizagem de residentes multiprofissionais relacionam-se à construção do aprender e fazer no trabalho coletivo, por meio da compreensão do papel do residente, a importância

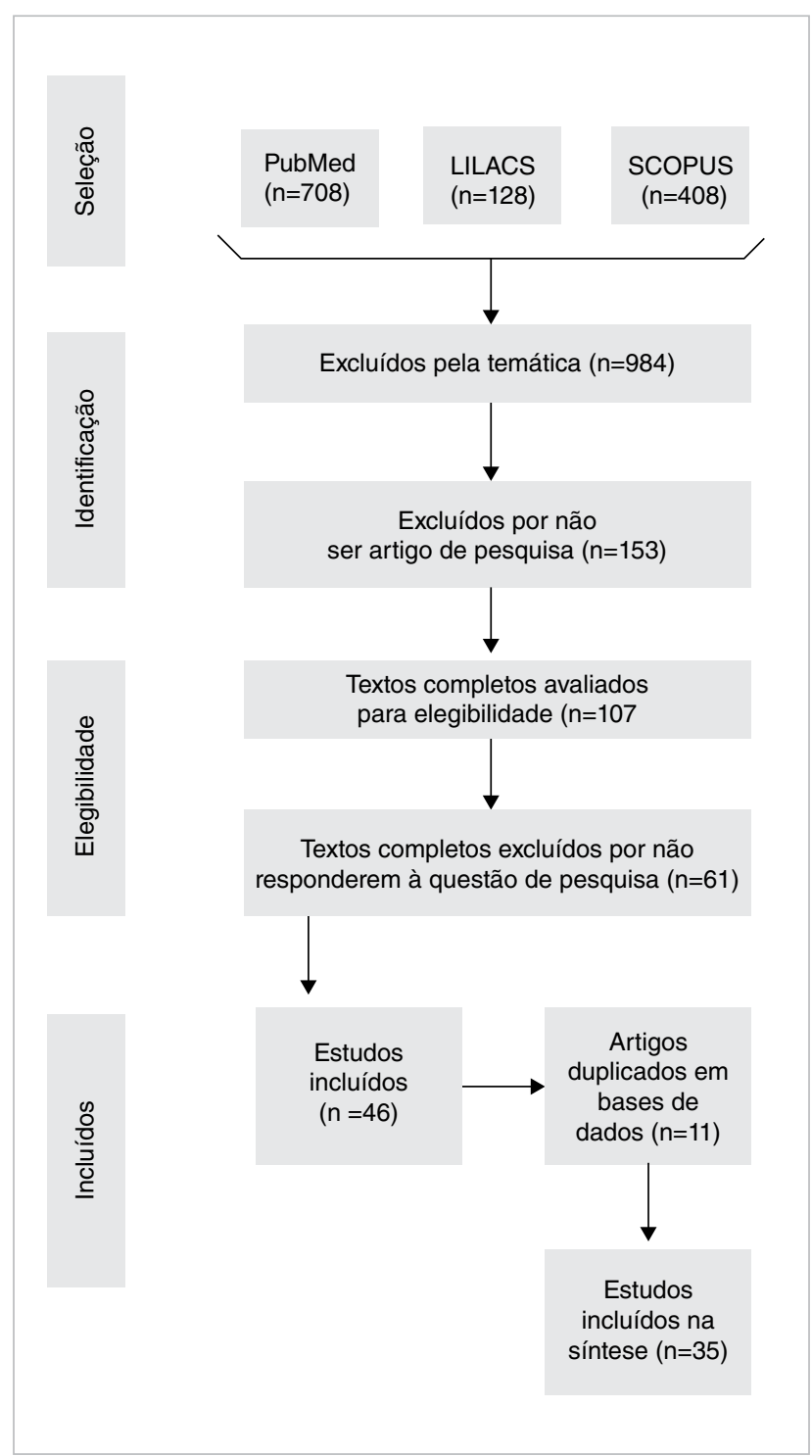

Figura 1: Processo de seleção amostral das publicações sobre os fatores que influenciam no ensino-aprendizagem de residentes multiprofissionais em saúde nas bases de dados nacionais e internacionais.

Tabela 1: Caracterização geral dos 35 artigos analisados, 2018.

\begin{tabular}{|l|c|c|}
\hline \multicolumn{2}{|c|}{$\mathbf{n}$} & $\%$ \\
\hline Procedência do primeiro autor & & \\
\hline Sudeste/Brasil & 13 & 37,14 \\
\hline Sul/Brasil & 10 & 28,57 \\
\hline Nordeste/Brasil & 7 & 20,00 \\
\hline Centro-Oeste/Brasil & 4 & 11,42 \\
\hline Canadá & 1 & 2,85 \\
\hline Delineamento de pesquisa & & \\
\hline Qualitativo & 28 & 80,00 \\
\hline Quantitativo & 5 & 14,28 \\
\hline Quanti-qualitativo & 2 & 5,71 \\
\hline Nível de Evidência & & \\
\hline Significado/experiência (CIV) NE 2 & 30 & 85,71 \\
\hline Diagnóstico (CII) NE 6 & 3 & 8,57 \\
\hline Prognóstico/etiologia (CIII) NE 4 & 2 & 5,71 \\
\hline Total & 35 & 100 \\
\hline
\end{tabular}


Tabela 2: Amostra das evidências encontradas segundo código utilizado, autores e título.

\begin{tabular}{|c|c|}
\hline Referência & Título \\
\hline Silva et al. ${ }^{1}$ & $\begin{array}{l}\text { Educação permanente em saúde a partir de profissionais } \\
\text { de uma residência multidisciplinar: estudo de caso. }\end{array}$ \\
\hline Bezerra et al. ${ }^{3}$ & $\begin{array}{l}\text { Avaliação de programas de formação profissional em } \\
\text { saúde: construção e validação de indicadores. }\end{array}$ \\
\hline Casanova et al..$^{5}$ & $\begin{array}{l}\text { Formação para o trabalho em equipe na residência } \\
\text { multiprofissional em saúde. }\end{array}$ \\
\hline Fernandes et al. ${ }^{6}$ & $\begin{array}{l}\text { Sofrimento e prazer no processo de formação de } \\
\text { residentes multiprofissionais em saúde. }\end{array}$ \\
\hline Lunas Junior et al. ${ }^{7}$ & $\begin{array}{l}\text { Posicionamento dos Coordenadores da Atenção Básica } \\
\text { Sobre Egressos da Residência Multiprofissional em Saúde } \\
\text { da Família. }\end{array}$ \\
\hline Santos et al. ${ }^{8}$ & $\begin{array}{l}\text { Residência Multiprofissional em Saúde da Família: } \\
\text { concepção de profissionais de saúde sobre a atuação do } \\
\text { nutricionista. }\end{array}$ \\
\hline Sanches et al. ${ }^{9}$ & $\begin{array}{l}\text { Burnout e Qualidade de Vida em uma Residência } \\
\text { Multiprofissional: um estudo longitudinal de dois anos. }\end{array}$ \\
\hline Goulart et al..$^{10}$ & $\begin{array}{l}\text { Perfil sociodemográfico e acadêmico dos residentes } \\
\text { multiprofissionais de uma universidade pública. }\end{array}$ \\
\hline Carvalho et al. ${ }^{11}$ & $\begin{array}{l}\text { Prevalência e fatores associados aos transtornos mentais } \\
\text { comuns em residentes médicos e da área multiprofissional. }\end{array}$ \\
\hline Guido et al. ${ }^{12}$ & $\begin{array}{l}\text { Síndrome de Burnout em residentes multiprofissionais de } \\
\text { uma universidade pública. }\end{array}$ \\
\hline Landim et al. ${ }^{13}$ & $\begin{array}{l}\text { Residência multiprofissional em saúde da família: vivência } \\
\text { hospitalar dos enfermeiros multiprofissional. }\end{array}$ \\
\hline
\end{tabular}
estratégia de educação permanente em saúde da família: o estudo do caso de Sobral, CE.

Rossoni ${ }^{15}$

Ferreira et al. ${ }^{16}$

Domingos et al. ${ }^{17}$

Nascimento \&

Oliveira $^{18}$

Silva et al. ${ }^{19}$

Scherer et al. ${ }^{20}$

Dallegrave et al. ${ }^{21}$

Conrad et al.22

Nordi \& Aciole ${ }^{23}$

Dias et al. ${ }^{24}$

Silva et al. ${ }^{25}$
Residência na atenção básica à saúde em tempos líquidos.

Trabalho em equipe multiprofissional: a perspectiva dos residentes médicos em saúde da família.

Potencialidades da Residência Multiprofissional em Saúde da Família: o olhar do trabalhador de saúde.

Competências profissionais e o processo de formação na Residência Multiprofissional em Saúde da Família e Comunidade

Perception of the residents about their performance in the multidisciplinary residency program.

A construção da interdisciplinaridade no trabalho da

No olho do furacão, na ilha da fantasia: a invenção da residência multiprofissional em saúde.

Participants' perceptions of a multidisciplinary training program for graduate and postgraduate students in drug use management and policy.

Apoio matricial: uma experiência da residência multiprofissional em saúde.

A tutoria no processo de ensino-aprendizagem no contexto da formação interprofissional em saúde.

Residência multiprofissional como espaço intercessor para a educação permanente em saúde. Equipe de Saúde da Família.
Fatores que influenciam no ensino-aprendizagem de residentes multiprofissionais em saúde

Planejamento e execução dos espaços formativos, como as oficinas, tutorias, preceptorias e seminários

Desenvolvimento dos processos avaliativos

Compreensão do papel do residente; interação entre as diferentes profissões; pactuação das atividades entre as instituições de ensino, serviços de saúde e de gestão; desenvolvimento das ações de Educação Permanente em Saúde; planejamento e execução dos espaços formativos, como as oficinas, tutorias, preceptorias e seminários.

Compreensão do papel do residente; interação entre as diferentes profissões; pactuação das atividades entre as instituições de ensino, serviços de saúde e de gestão; relação entre a teoria e a prática.

Compreensão do papel do residente; relação entre a teoria e a prática.

Compreensão do papel do residente; interação entre as diferentes profissões; pactuação das atividades entre as instituições de ensino, serviços de saúde e de gestão.

Compreensão do papel do residente

Compreensão do papel do residente

Compreensão do papel do residente

Compreensão do papel do residente

Formação dos apoiadores pedagógicos (preceptores e tutores); relação entre a teoria e a prática

Formação dos apoiadores pedagógicos (preceptores e tutores); formulação das estratégias de aprendizagem; planejamento e execução dos espaços formativos, como as oficinas, tutorias, preceptorias e seminários.

Formação dos apoiadores pedagógicos (preceptores e tutores)

Formação dos apoiadores pedagógicos (preceptores e tutores)

Interação entre as diferentes profissões; pactuação das atividades entre as instituições de ensino, serviços de saúde e de gestão.

Interação entre as diferentes profissões; pactuação das atividades entre as instituições de ensino, serviços de saúde e de gestão.

Interação entre as diferentes profissões; pactuação das atividades entre as instituições de ensino, serviços de saúde e de gestão.

Interação entre as diferentes profissões; pactuação das atividades entre as instituições de ensino, serviços de saúde e de gestão; relação entre a teoria e a prática.

Interação entre as diferentes profissões; pactuação das atividades entre as instituições de ensino, serviços de saúde e de gestão.

Interação entre as diferentes profissões; pactuação das atividades entre as instituições de ensino, serviços de saúde e de gestão.

Interação entre as diferentes profissões; pactuação das atividades entre as instituições de ensino, serviços de saúde e de gestão; desenvolvimento das ações de Educação Permanente em Saúde.

Interação entre as diferentes profissões; pactuação das atividades entre as instituições de ensino, serviços de saúde e de gestão.

Relação entre a teoria e a prática; desenvolvimento das ações de Educação Permanente em Saúde. 
Tabela 2: Continuação.

\begin{tabular}{|c|c|c|}
\hline Referência & Título & $\begin{array}{l}\text { Fatores que influenciam no ensino-aprendizagem } \\
\text { de residentes multiprofissionais em saúde }\end{array}$ \\
\hline Jesus \& araújo 26 & $\begin{array}{l}\text { Politização e formação em serviço: significados e } \\
\text { sentidos atribuídos pelos residentes em uma residência } \\
\text { multiprofissional em saúde mental na Bahia. }\end{array}$ & $\begin{array}{l}\text { Pactuação das atividades entre as instituições de ensino, } \\
\text { serviços de saúde e de gestão. }\end{array}$ \\
\hline Martins et al. ${ }^{27}$ & $\begin{array}{l}\text { Implementação de residência multiprofissional em saúde } \\
\text { de uma universidade federal: trajetória histórica. }\end{array}$ & $\begin{array}{l}\text { Pactuação das atividades entre as instituições de ensino, } \\
\text { serviços de saúde e de gestão. }\end{array}$ \\
\hline Manho et al. ${ }^{28}$ & $\begin{array}{l}\text { Reflexões sobre a prática do residente terapeuta } \\
\text { ocupacional na estratégia saúde da família no município } \\
\text { de São Carlos. }\end{array}$ & $\begin{array}{l}\text { Desenvolvimento das ações de Educação Permanente } \\
\text { em Saúde }\end{array}$ \\
\hline Brites et al. ${ }^{29}$ & $\begin{array}{l}\text { "A gente vai aprendendo": o apoio matricial na estratégia } \\
\text { de saúde da família em um programa de residência } \\
\text { multiprofissional integrada no interior do Rio Grande do } \\
\text { Sul, Brasil. }\end{array}$ & Relação entre a teoria e a prática \\
\hline Landim et al. ${ }^{30}$ & $\begin{array}{l}\text { Vivência clínica hospitalar: significados para enfermeiros } \\
\text { residentes em Saúde da Família. }\end{array}$ & Relação entre a teoria e a prática \\
\hline Honda \& Chirelli ${ }^{31}$ & $\begin{array}{l}\text { Residência Multiprofissional em Saúde: formação com } \\
\text { metodologias ativas de ensino-aprendizagem. }\end{array}$ & $\begin{array}{l}\text { Relação entre a teoria e a prática; Formulação das } \\
\text { estratégias de aprendizagem }\end{array}$ \\
\hline Miranda Neto et al. ${ }^{32}$ & $\begin{array}{l}\text { Residências multiprofissionais em saúde: análise } \\
\text { documental de projetos político-pedagógicos. }\end{array}$ & $\begin{array}{l}\text { Formulação das estratégias de aprendizagem; Relação } \\
\text { entre a teoria e a prática }\end{array}$ \\
\hline Melo et al. ${ }^{33}$ & $\begin{array}{l}\text { Problematizando a residência multiprofissional em } \\
\text { oncologia: protocolo de ensino prático na perspectiva de } \\
\text { residentes de enfermagem. }\end{array}$ & $\begin{array}{l}\text { Formulação das estratégias de aprendizagem; Relação } \\
\text { entre a teoria e a prática }\end{array}$ \\
\hline Lobato et al. ${ }^{34}$ & A dimensão política na formação dos profissionais de saúde. & $\begin{array}{l}\text { Formulação das estratégias de aprendizagem; planejamento } \\
\text { e execução dos espaços formativos, como as oficinas, } \\
\text { tutorias, preceptorias e seminários; Desenvolvimento dos } \\
\text { processos avaliativos }\end{array}$ \\
\hline Lima \& Santos ${ }^{35}$ & $\begin{array}{l}\text { Formação de psicólogos em residência multiprofissional: } \\
\text { transdisciplinaridade, núcleo profissional e saúde mental. }\end{array}$ & $\begin{array}{l}\text { Planejamento e execução dos espaços formativos, como as } \\
\text { oficinas, tutorias, preceptorias e seminários. }\end{array}$ \\
\hline Salvador et al. ${ }^{36}$ & $\begin{array}{l}\text { Construindo a multiprofissionalidade: um olhar sobre } \\
\text { a residência multiprofissional em saúde da família e } \\
\text { comunidade. }\end{array}$ & $\begin{array}{l}\text { Planejamento e execução dos espaços formativos, como as } \\
\text { oficinas, tutorias, preceptorias e seminários. }\end{array}$ \\
\hline Vasconcelos et al. ${ }^{37}$ & $\begin{array}{l}\text { Avaliação de programas de residência multiprofissional } \\
\text { em saúde da família por indicadores. }\end{array}$ & Desenvolvimento dos processos avaliativos \\
\hline
\end{tabular}

da formação dos apoiadores pedagógicos e a interação entre as diferentes profissões; a integração ensino-serviço, a partir da pactuação das atividades entre as instituições de ensino e dos serviços de saúde e de gestão, a relação entre a teoria e a prática e o desenvolvimento das ações de Educação Permanente em Saúde; e, por fim, a organização didática pedagógica a partir da formulação das estratégias de aprendizagem; do planejamento e execução dos espaços formativos; e, os processos avaliativos. Para melhor compreensão, estes resultados estão esquematizados na Figura 2.

\section{DISCUSSÃO}

\section{Construção do aprender e fazer no coletivo}

Os Programas de Residências Multiprofissionais (PRMS) estão em plena expansão no território brasileiro, tornando-se uma estratégia de formação em saúde que busca a integração entre o ensino e o serviço e o fortalecimento da qualidade da atenção à saúde da população. Com o aumento expressivo da demanda de candidatos aos PRMS e da ampla criação dos mesmos em diferentes áreas profissionais, distribuídas em Programas no Brasil, observa-se um crescimento expressivo de produções acerca da temática nos últimos anos. A procura cada vez maior por essa especialização em saúde pode ser justificada pela busca de qualificação para inserção no mercado de trabalho ${ }^{39}$.

Com as reformulações no campo da saúde, os PRMS ofertam a possibilidade de aprender e fazer no coletivo, por meio da experiência da multiprofissionalidade. A interação entre diferentes profissões foi evidenciada como uma possibilidade para aprender no e com o coletivo, por meio da troca de conhecimentos ${ }^{15,29}$. O trabalho cooperado é constituído como uma possibilidade de lidar para favorecer o exercício de habilidades e atitudes, além do respeito pelas diferenças a partir de um movimento de busca, diálogo, desafio, comprometimento e responsabilidade ${ }^{5,6}$.

Essa interação profissional ofertada pela Residência em Saúde oferta a reflexão teórica e a troca de conhecimentos frente a integralidade no trabalho em saúde ${ }^{17,19}$. Ações como visita domiciliar, grupos educativos, espaços de clínicas em equipe e as interconsultas, são momentos de integração que podem impulsionar essa interação e, consequentemente, a elaboração de planos terapêuticos na perspectiva da integralidade $e^{8,20}$.

Para tanto, ainda há desafios que permeiam o aprender e fazer nos PRMS e, consequentemente, dificultam o trabalho coletivo, como o ingresso de profissionais oriundos de uma formação estritamente 
clínica e uniprofissional ${ }^{20,29}$. As limitações na graduação influenciam na experiência dos residentes, para tanto, ações que vem sendo desenvolvidas como os Projetos Pró-saúde e PET-saúde são estratégias que podem fortalecer e provocar a reformulação da formação profissional para que o residente não seja impactado pelo novo.

Além disso, demais atividades podem ser desenvolvidas durante o processo de ensino-aprendizagem dos residentes, como espaços que o façam (re)construir a sua identidade profissional a fim de facilitar o entendimento e a aplicação de novas perspectivas e ampliação do campo de valores da sua prática profissional ${ }^{23}$. A análise dos estudos evidenciou que há a falta de entendimento e reconhecimento dos princípios do papel do residente. Com base nisso, questiona-se: se por vezes os residentes não reconhecem a sua própria identidade profissional como os demais membros das equipes de saúde poderão compreender o papel do residente? Essas questões implicam diretamente motivação a formação e no trabalho $0^{6,7,8,20,27}$.

Para isso, faz-se importante a formação de apoiadores pedagógicos para atuarem como preceptores e tutores, especialmente o preceptor por obter um papel de facilitador do processo pedagógico dos residentes nos serviços de saúde, na medida em que se encontra em interação constante com estes. Essa interação é permeada por relações, ora profissional, ora pessoal, advindas do convívio diário no âmbito do trabalho, onde trabalhadores especialistas compartilham saberes e vivências com residentes, em sua maioria, recém-formados e inexperientes ${ }^{33}$.

Para dar início a uma relação produtiva, é essencial que os envolvidos se interessem e estejam envolvidos com o planejamento da residência, pois, assim, poderá articular os serviços de saúde de acordo com as necessidades do usuário e a aprendizagem do residente ${ }^{22,24}$. Entretanto, associar as práticas assistenciais às de ensino é uma atividade complexa, especialmente ao se retratar aos PRMS, visto que o preceptor e, também, o tutor, necessita integrar com residentes oriundos de diferentes categorias profissionais e com necessidades de aprendizagem distintas. Nesse sentido, faz-se premente que os preceptores possam preparo pedagógico para auxiliar no pensar-agir reflexivo e crítico, auxiliando, dessa forma a práxis em saúde ${ }^{40}$.

\section{Integração ensino-serviço}

A integração ensino-serviço influencia no ensino e aprendizagem destes residentes. Evidenciou-se a importância das pactuações das atividades dos residentes com os envolvidos na formação (gestores, preceptores, usuários e/ou residentes), visto que as atividades desenvolvidas pelos residentes são realizadas nos serviços de saúde. Reconhece-se que a interação entre os residentes e os trabalhadores de saúde contribui para a melhoria da qualidade da assistência prestada, favorece a integralidade da assistência e possibilita a inclusão de diversas categorias profissionais que não atuavam anteriormente nos serviços de saúde $e^{5,13,17,28}$. Para tanto, o trabalho em equipe e o desenvolvimento das atividades podem sofrer interferências pela inserção dos residentes em cenários de fragilidade das unidades de saúde, sendo que os residentes assumem as escalas de serviço, principalmente por falta de trabalhadores ${ }^{6,15,29}$.

Todavia, no momento em que houver cooperação recíproca e planejamento de atividades entre os gestores, residentes, programa de residência e profissionais das Unidades de Saúde, por meio da troca de conhecimentos e do trabalho coletivo, a interação



Figura 2: Síntese dos resultados evidenciados na pesquisa de revisão. 
entre trabalhadores e residentes tenderá a possibilitar a reflexão teórica sobre o trabalho cotidiano $0^{7,17,20}$. Além de ser uma possibilidade de efetivar e qualificar as ações de EPS, como o matriciamento na saúde, a criação de espaços efetivos de discussões de casos, clínicas em equipe (interconsultas), impulsionar a elaboração de planos terapêuticos em equipe e a intervenção conjunta e ações de educação permanente $e^{5,20,28,36}$.

Dentro deste contexto, faz-se necessário a colaboração e articulação entre os locais de formação, ensino e serviço ${ }^{30}$. As instituições que se corresponsabilizam pela formação em saúde, especialmente ao que diz respeito aos PRMS, necessitam compreender os objetivos pedagógicos do Programa, além de expor as expectativas, preocupações e dúvidas tanto dos residentes, quanto dos trabalhadores, gestores e demais envolvidos, com vistas a promover um espaço tranquilo e propício ao aprendizado ${ }^{33}$.

Com base nesse contexto, o relacionamento entre a teoria e a prática foi um aspecto evidenciado na revisão, sendo que a dificuldade em relacionar os conteúdos aprendidos à realidade apresentada evidencia a importância de um investimento no permanente diálogo da ação (o que fazer) com o interlocutor (situação) $)^{30}$. Esse aspecto pode ser consequência do despreparo inicial para lidar com as variadas demandas que exigem dos residentes maior esforço e disposição, uma vez que a residência corresponde ao início de uma carreira profissional e, por vezes, há limitações na graduação $0^{9,20,28}$.

A formação nos serviços de saúde prima pelo desenvolvimento de práticas formativas com base na experimentação e em ações de EPS. Há exemplo disto, uma pesquisa desenvolvida em um PRMS apontou que existem melhores práticas de EPS dos preceptores em acolher os residentes no serviço onde estes serão admitidos, proporcionando uma segurança aos residentes e uma melhor relação entre os atores envolvidos ${ }^{1}$.

\section{Organização didática pedagógica}

Ademais, conforme a análise das evidências, outro aspecto que influencia na formação dos residentes é a organização didática pedagógica deste processo de aprendizagem nos Programas. As estratégias de ensino utilizadas pelos PRMS necessitam contribuir para que o residente seja ator de seu próprio processo buscando de ativamente novas informações sobre o as questões de saúde, tanto regionais quanto nacionais, o que o levará a problematizar frente ao cenário visando possíveis alternativas de intervenção² .

As atividades de ensino-aprendizagem precisam estabelecer um diálogo permanente com os princípios do SUS para o alcance da integralidade e o trabalho cooperado entre os diversos núcleos de saberes ${ }^{22,36,38}$. O planejamento dessas atividades suscita o uso de artifícios que possibilitem a troca de saberes e consiga valorizar as diferentes áreas de conhecimentos ${ }^{22,36}$. Como exemplo, pode-se apontar a utilização de metodologias ativas a fim de proporcionar o aluno a problematização da realidade ${ }^{31}$.
Acrescenta-se ainda, que as avaliações frente ao processo pedagógico necessitam abranger aspectos que considere as experiências vivenciadas no decorrer dos cenários de prática bem como, o crescimento profissional do residente. Compreendendo, dessa forma, sua aprendizagem teórica e científica, sua capacidade de reflexão crítica, tomada de decisão, além dos aspectos práticos ${ }^{33}$.

Evidencia-se, assim, a emergência de propostas de avaliação que considerem as tanto as competências referentes à área do conhecimento (núcleo) como as vivências multiprofissionais nos cenários de práticas (campo), não se restringindo somente a atividades teórico-conceituais comumente adotadas pelos programas ${ }^{33}$. Para isso, o avançado aporte de recursos tecnológicos e a estrutura física à disposição do programa pode possibilitar o aprimoramento do conhecimento em saúde dos residentes ${ }^{33}$.

Conforme explícito na análise dos artigos, a organização didática pedagógica dos espaços como oficinas, tutorias, preceptorias e seminários de campo e de núcleo é um fator importante a ser configurado, pois estes podem ser os momentos de discussão e trocas de experiências dos residentes com outros profissionais. Sendo que, esses espaços, podem tencionar a construção do conhecimento, as articulações com as outras áreas do saber o trabalho coletivo e a oportunidade de novos conhecimentos, acerca de outras áreas ${ }^{16,17,19}$.

\section{CONSIDERAÇÕES FINAIS}

Em relação à contribuição dessa revisão para a pesquisa e o ensino, observa-se que os Programas de Residência Multiprofissionais em Saúde vêm conquistando espaços no cenário de pesquisas e de qualificação profissional no país. Por se apresentarem como uma importante estratégia para formação profissional, este estudo contribui para a assistência em saúde no que se refere ao processo pedagógico dos PRMS a fim de auxiliar no aprimoramento do cenário real. Cumpre assinalar que este estudo possui limitações referentes à estratégia de busca, visto que não há descritores cadastrados no DECS ao que se refere aos PRMS, para isso, buscou-se utilizar palavras-chave, o que pode ter acarretado a não inclusão de alguns estudos sobre a temática.

Ademais, observa-se o elevado número de estudos exploratórios e descritivos, que vão ao encontro da preocupação em compreender a realidade a partir de uma perspectiva analítica mais ampla dos fenômenos estudados; corroborando ao considerar que os fenômenos da formação profissional na área da saúde são complexos e, em geral, necessitam de uma perspectiva baseada em abordagens que possam contribuir para um entendimento generalizado e crítico sobre esse processo. Vislumbra-se, por fim, a importância do desenvolvimento de pesquisas que considerem diferentes contextos, abordagens e populações especialmente com o desenvolvimento de estudos com abordagens intervencionistas como as pesquisas participativas, assim como métodos mistos os quais são escassos em relação a essa temática. 


\section{REFERÊNCIAS}

1. Silva CT, Terra MG, Camponogara S, Kruse MHL, Roso CC, Xavier MS. Educação permanente em saúde a partir de profissionais de uma residência multidisciplinar: estudo de caso. Rev Gaúcha Enferm. 2014;35(3):49-54.

http://dx.doi.org/10.1590/1983-1447.2014.03.44512

2. Fiorano AMM, Guarnieri AP. Residência multiprofissional em saúde: tem valido a pena? ABCS Health Sci. 2015; 40(3):366-9. https://dx.doi.org/10.7322/abcshs.v40i3.823

3. Bezerra TCA, Falcão MLP, Goes PSA, Felisberto E. Avaliação de programas de formação profissional em saúde: construção e validação de indicadores. Trab Educ Saúde. 2016;14(2):445-72. http://dx.doi.org/10.1590/1981-7746-sip00111

4. Canabarro JL, Siqueira DF, Terra MG. A inserção dos profissionais integrantes das residências multiprofissionais em serviços de saúde: revisão de literatura. Rev Soc Hum. 2016;29(3):121-28. http://dx.doi.org/10.5902/2317175824168

5. Casanova IA, Batista NA, Ruiz-Moreno L. Formação para o trabalho em equipe na residência multiprofissional em saúde. ABCS Health Sci. 2015;40(3)229-33.

https://dx.doi.org/10.7322/abcshs.v40i3.800

6. Fernandes MNS, Beck CLC, Weiller TH, Viero V, Freitas PH, Prestes FC. Sofrimento e prazer no processo de formação de residentes multiprofissionais em saúde. Rev Gaúcha Enferm. 2015;36(4):90-7.

http://dx.doi.org/10.1590/1983-1447.2015.04.50300

7. Lunas Junior FJ, Barreto RMA, Vasconcelos MIO. Posicionamento dos coordenadores da Atenção Básica sobre egressos da residência multiprofissional em Saúde da Família. Rev Bras Ciênc Saúde. 2014;18(4):325-32.

8. Santos IG, Batista NA, Devincenzi MU. Residência Multiprofissional em Saúde da Família: concepção de profissionais de saúde sobre a atuação do nutricionista. Interface. 2015;19(53):349-60. http://dx.doi.org/10.1590/1807-57622014.0330

9. Sanches VS, Ferreira PM, Veronez AV, Koch R, Souza AS, Cheade MFM, et al. Burnout e Qualidade de Vida em uma Residência Multiprofissional: um Estudo longitudinal de dois anos. Rev Bras Educ Méd. 2016;40(3):430-6. http://dx.doi.org/10.1590/1981-52712015v40n3e01022015

10. Goulart CT, Silva RM, Bolzan MEO, Guido LA. Perfil sociodemográfico e acadêmico dos residentes multiprofissionais de uma universidade pública. Rev Rene. 2012;13(1):178-86.

11. Carvalho CN, Melo-Filho DA, Carvlho JAG, Amorim ACG. Prevalência e fatores associados aos transtornos mentais comuns em residentes médicos e da área multiprofissional. J Bras Psiquiatr. 2013;62(1):38-45.

http://dx.doi.org/10.1590/S0047-20852013000100006

12. Guido LA, Silva RM, Goulart CT, Bolzan MEO, Lopes LFD. Síndrome de Burnout em residentes multiprofissionais de uma universidade pública. Rev Esc Enferm USP. 2012;46(6):1477-83. http://dx.doi.org/10.1590/S0080-62342012000600027

13. Landim SA, Silva GTR, Batista NA. Residência multiprofissional em saúde da família: vivência hospitalar dos enfermeiros multiprofissional. Rev Baiana Enferm. 2012;26(1):375-86. http://dx.doi.org/10.18471/rbe.v26i1.6050

14. Pagani R, Andrade LOM. Preceptoria de território, novas práticas e saberes na estratégia de educação permanente em saúde da família: o estudo do caso de Sobral, CE. Saúde Soc. 2012;21(supl.1):94-106.

http://dx.doi.org/10.1590/S0104-12902012000500008
15. Rossoni E. Residência na atenção básica à saúde em tempos líquidos. Physis. 2015;25(3):1011-31. http://dx.doi.org/10.1590/S0103-73312015000300017

16. Ferreira RC, Varga CRR, Silva RF. Trabalho em equipe multiprofissional: a perspectiva dos residentes médicos em saúde da família. Ciênc Saúde Coletiva. 2009;14(supl.1):1421-8. http://dx.doi.org/10.1590/S1413-81232009000800015

17. Domingos CM, Nunes EFPA, Carvalho BG. Potencialidades da Residência Multiprofissional em Saúde da Família: o olhar do trabalhador de saúde. Interface. 2015;19(55):1221-32. http://dx.doi.org/10.1590/1807-57622014.0653

18. Nascimento DDG, Oliveira MAC. Competências profissionais e o processo de formação na Residência Multiprofissional em Saúde da Família e Comunidade. Saúde Soc. 2010;19(4):814-27. http://dx.doi.org/10.1590/S0104-12902010000400009

19. Silva JC, Contim D, Ohl RIB, Chavaglia SRR, Amaral EMS. Perception of the residents about their performance in the multidisciplinary residency program. Acta Paul Enferm. 2015;28(2):132-38.

http://dx.doi.org/10.1590/1982-0194201500023

20. Scherer MDA, Pires DEP, Jean R. A construção da interdisciplinaridade no trabalho da Equipe de Saúde da Família. Ciênc Saúde Coletiva. 2013;18(11):3203-12.

http://dx.doi.org/10.1590/S1413-81232013001100011

21. Dallegrave D, Kruse MHL. No olho do furacão, na ilha da fantasia: a invenção da residência multiprofissional em saúde. Interface. 2009;13(28):213-26.

http://dx.doi.org/10.1590/S1414-32832009000100018

22. Conrad P, Sketris I, Langille-Ingram E. Participants' perceptions of a multidisciplinary training program for graduate and postgraduate students in drug use management and policy. Am J Pharm Educ. 2013;77(5):102.

http://dx.doi.org/10.5688/ajpe775102

23. Nordi ABA, Aciole GG. Apoio matricial: uma experiência da residência multiprofissional em saúde. Trab Educ Saúde. 2017; 15(2):485-500.

http://dx.doi.org/10.1590/1981-7746-sol00053

24. Dias IMAV, Pereira AK, Batista SHSS, Casanova IA. A tutoria no processo de ensino-aprendizagem no contexto da formação interprofissional em saúde. Saúde Debate. 2016:40(111):257-67. http://dx.doi.org/10.1590/0103-1104201611120

25. Silva CT, Terra MG, Kruse MHL, Camponogara S, Xavier MS Residência multiprofissional como espaço intercessor para a educação permanente em saúde. Texto Contexto Enferm. 2016;25(1):e2760014 http://dx.doi.org/10.1590/0104-0707201600002760014

26. Jesus ML, Araújo D. Politização e formação em serviço: significados e sentidos atribuídos pelos residentes em uma residência multiprofissional em saúde mental na Bahia. Psicol Teor Prat. 2011;13(3):67-80.

27. Martins GDM, Caregnato RCA, Barroso VLM, Ribas DCP. Implementação de residência multiprofissional em saúde de uma universidade federal: trajetória histórica. Rev Gaúcha Enferm. 2016;37(3):e57046.

http://dx.doi.org/10.1590/1983-1447.2016.03.57046

28. Manho F, Soares LBT, Nicolau SM. Reflexões sobre a prática do residente terapeuta ocupacional na estratégia saúde da família no município de São Carlos. Rev Ter Ocup USP. 2013;24(3):233-41. http://dx.doi.org/10.11606/issn.2238-6149.v24i3p233-241 
29. Brites LS, Weiller TH, Silveira D, Françóis APW, Rosa FMello, Righi LB. "A gente vai aprendendo": o apoio matricial na estratégia de saúde da família em um programa de residência multiprofissional integrada no interior do Rio Grande do Sul, Brasil. Saúde Debate. 2014;38(101):285-95.

http://dx.doi.org/10.5935/0103-1104.20140026

30. Landim SA, Batista NA, Silva GTR. Vivência clínica hospitalar: significados para enfermeiros residentes em Saúde da Família. Rev Bras Enferm. 2010;63(6):913-20.

http://dx.doi.org/10.1590/S0034-71672010000600007

31. Honda K, Chirelli MQ. Residência Multiprofissional em Saúde: formação com metodologias ativas de ensino-aprendizagem. Indag Didac. 2015;7(3):49-61.

32. Miranda Neto MV, Leonello VM, Oliveira MAC. Residências multiprofissionais em saúde: análise documental de projetos político-pedagógicos. Rev Bras Enferm. 2015;68(4):586-93. http://dx.doi.org/10.1590/0034-7167.2015680403

33. Melo MC, Queluci GC, Gouvêa MV. Problematizando a residência multiprofissional em oncologia: protocolo de ensino prático na perspectiva de residentes de enfermagem. Rev Esc Enferm USP. 2014;48(4):706-14.

http://dx.doi.org/10.1590/S0080-623420140000400019

34. Lobato CP, Melchior R, Baduy RS. A dimensão política na formação dos profissionais de saúde. Physis. 2012;22(4):1273-91. http://dx.doi.org/10.1590/S0103-73312012000400002
35. Lima M, Santos L. Formação de psicólogos em residência multiprofissional: transdisciplinaridade, núcleo profissional e saúde mental. Psicol Ciênc Prof. 2012;32(1):126-41. http://dx.doi.org/10.1590/S1414-98932012000100010

36. Salvador AS, Medeiros CS, Cavalcanti PB, Carvalho RN Construindo a multiprofissionalidade: um olhar sobre a residência multiprofissional em saúde da família e comunidade. Rev Bras Ciênc Saúde. 2011;15(3):329-38.

37. Vasconcelos MIO, Souza FL, Lira GV, Dias MSA, Silva GSN. Avaliação de programas de residência multiprofissional em saúde da família por indicadores. Trab Educ Saúde. 2015;13(supl.2):53-77. http://dx.doi.org/10.1590/1981-7746-sip00080

38. Stillwell. Asking compelling, clinical questions. In: Melnyk BM Fineout-Overholt E. Evidence-based practice in nursing \& healthcare. A guide to best practice. Philadelphia: Wolters Kluwer, Lippincott Williams \& Wilkins; 2011.

39. Sarmento LF, França T, Medeiros KR, Santos MR, Ney MS. A distribuição regional da oferta de formação na modalidade Residência Multiprofissional em Saúde. Saúde Debate. 2017;41(113):415-24

http://dx.doi.org/10.1590/0103-1104201711306

40. Ribeiro KRB, Prado ML. A prática educativa dos preceptores nas residências em saúde: um estudo de reflexão. Rev Gaúcha Enferm. 2013:34(4):161-5.

http://dx.doi.org/10.1590/1983-1447.2014.01.43731 